\title{
Feasibility of Long-Term Continuous EEG Monitoring During the First Days of Life in Preterm Infants: An Automated Quantification of the EEG Activity
}

\author{
EVA M. SCHUMACHER, ASBJORN S. WESTVIK, PÅL G. LARSSON, ROLF LINDEMANN, JOSTEIN WESTVIK, \\ AND TOM A. STIRIS
}

\begin{abstract}
Department of Pediatrics [E.M.S., R.L., T.A.S.], Oslo University Hospital, Ullevaal, 0407 Oslo, Norway; Faculty of Medicine [A.S.W.], University of Oslo, 0316 Oslo, Norway; Department of Neurology [P.G.L.], Department of Radiology [J.W.], Oslo University Hospital, Rikshospitalet, 0027 Oslo, Norway
\end{abstract}

\begin{abstract}
Long-term EEG monitoring (LTM) with several electrodes could be a useful tool for surveillance of the brain during the first critical days of life. This study aimed to assess the feasibility of multichannel LTM for automated analysis of EEG activity from d 1 to 3 using eight electrodes. Premature infants (GA $<31 \mathrm{wk} ; n=48)$ were continuously monitored for $3 \mathrm{~d}$. EEG monitoring for a total of $3257 \mathrm{~h}$ was successfully performed. Total absolute band power (tABP) was calculated per second. Artifacts were removed visually or by an algorithm removing the highest 5, 10, 15, and 20\% tABPs. NS difference was found between the trends of visually edited and $5 \%$ mathematically trimmed data. Two groups were compared ( $24 \leq \mathrm{GA}<28 \mathrm{wk}$ and $28 \leq \mathrm{GA}<31 \mathrm{wk})$ using the median of tABP for all frequency bands per day. The results showed that $\mathrm{tABP}$ differed between groups. The changes of tABP d 1-3 were equal in both groups. Automatically assessed LTM confirms that the EEG activity depends on GA. However, it reveals that the early changes (d 1-3) are independent of GA. The study demonstrates the feasibility of multichannel LTM and the possibility of developing automated EEG analyses. (Pediatr Res 69: 413-417, 2011)
\end{abstract}

$\mathrm{P}$ reterm and immature infants are at great risk for neurological disabilities $(1,2)$. An applicable method for continuous EEG monitoring and an automatic quantification of the global brain activity would probably be of great benefit for early detection of pathological processes. This could help in early prediction of long-term outcome and facilitate early counseling of parents. It could also be a useful tool for early selection for neuroprotective interventions when these become available. Many NICUs monitor the brain activity by amplitude-integrated EEG (aEEG), a simplified single- or twochannel EEG monitoring providing continuous information on the brain activity from limited parts of the brain. Despite few channels, it has proved to be successful in early assessment of the brain maturation (3) and prediction of later outcome in preterm infants with intraventricular hemorrhage (IVH) or white matter disease (4-6). The value of conventional EEG recording in neonatal intensive care is also well recognized. Many EEG features give important information on cerebral

Received July 21, 2010; accepted December 3, 2010.

Correspondence: Tom A. Stiris, M.D., Ph.D., Department of Pediatrics, Oslo University Hospital, Ullevaal, 0407 Oslo, Norway; e-mail: t.a.stiris@medisin.uio.no

Supported by Civitan Norge, UNI Foundation, and the Eastern Norway Regional Health Authority. maturation and brain functioning during the neonatal period (7-10).

Long-term EEG monitoring (LTM) of the immature infants applying many channels is not established in the NICU. One reason for the delay may be the assumed difficulties of the physical handling of the small vulnerable infants. Another problem is the lack of an algorithm for reliable EEG analyses and thus the dependency of the time-consuming visual editing for identifying and removing artifacts in a LTM (11-15). In addition, visual editing is a subjective method that requires high interpreter competence and experts may come to different conclusions (16).

We hypothesized that early multichannel LTM is feasible in the NICU setting without interfering with the handling and other types of surveillance of the premature infant. Furthermore, this procedure might add useful novel information on the brain in preterm infants during the first critical days of life. We also hypothesized that digital EEG recording could reveal the possibility of an automated quantification of the brain activity during a LTM. The aim of this study was a 72-h surveillance of the preterm brain using continuous multichannel EEG recording starting soon after birth and to develop a method for automated analysis of the continuous EEG activity.

\section{METHOD}

Subjects. Premature infants from $24+0$ wk to $30+6$ wk GA determined by prenatal ultrasound (US), without congenital anomalies, were included from the NICU, Department of Pediatrics, Oslo University Hospital, Ullevaal, during a 22 -mo period. The infants were divided into group 1 ( $24 \leq \mathrm{GA}<$ $28 \mathrm{wk})$ and group $2(28 \leq \mathrm{GA}<31 \mathrm{wk})$. Parents of eligible subjects were asked to participate. We aimed to start EEG monitoring within $12 \mathrm{~h}$ after delivery. The infants were enrolled consecutively after written informed consent. The study was approved by the Regional Committee for Medical Ethics and the Norwegian Data Protection Supervisor.

EEG monitoring. Eight EEG electrodes were placed according to the international 10-20 system in a reduced montage modified for the small head sizes of preterm infants. The EEG electrodes were placed at Fp1, P3, O1, T3 and $\mathrm{Fp} 2, \mathrm{P} 4, \mathrm{O} 2, \mathrm{~T} 4$ positions. The central electrodes commonly used in the

Abbreviations: ABP, absolute band power; FFT, fast Fourier transform; IVH, intraventricular hemorrhage; IntABP, natural logarithm of total ABP; LTM, long-term EEG monitoring; tABP, total ABP; tRBP, total relative band power; US, ultrasound 
reduced montage for neonates were replaced by the parietal electrodes allowing US access over the vertex. Eight-channel EEG was recorded through a digital EEG system using NicoletOne version 5.2 (CareFusion, CA). The conventional filtering of the EEG with a high-pass filter of $0.5 \mathrm{~Hz}$ and a low-pass filter of $70 \mathrm{~Hz}$ and a notch filter of $50 \mathrm{~Hz}$ was applied. The sampling rate was $256 \mathrm{~Hz}$ for digitization of the EEG signals. The EEG data were recorded online and stored directly onto a central hospital server.

Disposable electrodes with a diameter of $15 \mathrm{~mm}$ (Blue Sensor BRS-50 K Ambu ECG electrodes; Medicotest A/S, Ølstykke, Denmark) were applied and pasted to the skin. To get good contact between the electrodes and the skin, EEG abrasive skin preparation gel (Nuprep; D.O. Weaver \& CO, Aurora, CO) was gently rubbed by cotton sticks before attachment. For keeping the electrodes in place and getting free access to the scalp, a specially designed cap was developed. The cap was split in the frontal midline and closed by a Velcro, which made it simple to open for electrode checks and perform cerebral US during the monitoring. The electrode impedances were checked every $3 \mathrm{~h}$ or more frequently if needed. Failing electrodes were replaced to sustain an adequate EEG quality throughout the recording. The electrode leads were gathered and led through an opening on the top of the cap, which could be closed or opened by a purse string. Mean arterial blood pressure (MAP), heart rate ( $\mathrm{HR}), \mathrm{CO}_{2}$ tension, and oxygen saturation were simultaneously monitored (Hewlett Packard, Palo Alto, CA).

Cerebral US. Daily cranial US was performed during the EEG monitoring (Acuson Sequoia, Siemens, Germany). IVH was classified into four grades according to Papile et al. (17). In our study, infants with IVH > I were considered to have pathological US.

EEG analysis. The EEG recordings were edited visually by a pediatrician trained in EEG interpretation and a clinical neurophysiologist experienced in reading neonatal EEG. Unreadable epochs or epochs in the recordings with impedances $>40 \mathrm{k} \Omega$ in any channel at the end of the epochs were discarded. An impedance of $40 \mathrm{k} \Omega$ was selected as the impedance limit according to Ferree et al. (18). NicoletOne EEG Review software based on the fast Fourier transform (FFT) was used for trend analysis. Absolute band power (ABP) was calculated for each second from 1 to $30 \mathrm{~Hz}$ applying 50\% overlapping blocks as implemented in NicoletOne, linear detrending, and a Hamming window for the bipolar channels FP1-T3; T3-O1; FP2-T4; T4-O2; FP1-P3; P3-O1; FP2$\mathrm{P} 4 ; \mathrm{P} 4-\mathrm{O} 2$. $\mathrm{ABP}$ of a frequency band is defined as the integral of all the power values within its frequency range and expressed in $\mu \mathrm{V}^{2}$. The ABP values for each second in all the bands $(\delta, 1.0-4.0 ; \theta, 4.0-8.0 ; \alpha, 8.0-13.0$; and $\beta$, $13.0-30.0 \mathrm{~Hz}$ ) were exported to the SAS System (SAS Institute Inc, Cary, $\mathrm{NC}$ ). Total ABP (tABP) was calculated for each band as the sum of a band's $\mathrm{ABP}$ for all eight channels resulting in 4 tABPs $(\delta$-tABP, $\theta$-tABP, $\alpha$-tABP, and $\beta$-tABP). Total relative band power (tRBP) was also calculated for each band as the ratio of the band's tABP to the sum of all four bands' tABPs (i.e. $\delta$-tRBP $=\delta$-tABP $/ \delta$-tABP $+\theta$-tABP $+\alpha$-tABP $+\beta$-tABP) expressed as a percentage. Medians for tABP and tRBP were calculated per infant per day.

The median values of tABP $d 1-3$ were analyzed by four algorithms for automated trimming in addition to the visually edited method. The automated trimming implicated removal of $5,10,15$, or $20 \%$ of the highest tABP values in each patient, respectively. The trimmed methods were compared with the visually edited method.

Statistical analyses. ANOVA with repeated measurements using marginal means for the differences between the visually edited and trimmed methods (SPSS software version 16.0; SPSS) was applied to test for statistical difference between the methods. ANOVA with repeated measurements was also used to test for changes in tABP and tRBP $\mathrm{d} 1-3$ in group 1 and group 2. A $p \leq 0.05$ was considered statistically significant.

\section{RESULTS}

Fifty-three infants were initially included of which five were excluded. Three because of a short EEG recording time $<48 \mathrm{~h}$ (one was in need of acute surgery, one died during the first $24 \mathrm{~h}$, and one was withdrawn by the parents). In two infants, sufficient electrode contact was not obtained due to hair. According to our protocol, the hair could not be removed. Consequently, 48 infants were EEG monitored over a period of $3 \mathrm{~d}$.

Feasibility of the recording. The monitoring time of all the patients was $3257 \mathrm{~h}$, with a mean of $67.9 \pm 4.0 \mathrm{~h}$ per patient. The start of EEG monitoring varied from 2.5 to $11.2 \mathrm{~h}$ after birth with a median of $5.3 \mathrm{~h}$.
Forty-five infants had phototherapy during the EEG recording, which was without difficulties. Of the total recording time, $96 \%$ had respiratory support [conventional mechanical ventilation, high-frequency oscillatory ventilation (HFOV), and continuous positive airway pressure (CPAP)] and was successfully combined with the EEG monitoring. The handling of the babies during the EEG electrode placement was well tolerated without need to interrupt the placement. No abrupt changes in the blood gases, blood pressure, and HR were observed. In all cases, the skin tolerated the electrodes. No wounds, rashes, or other skin complications were reported.

$\boldsymbol{E} \boldsymbol{E} \boldsymbol{G}$ results. In this study, EEG was analyzed only in infants without cerebral pathology $(\mathrm{IVH} \leq \mathrm{I})$ during the first 3 days of life. Of the 48 monitored infants, six infants were diagnosed with IVH $>$ I. Consequently, 42 infants with normal cerebral US were included in the further analyses. None of them received sedative drugs.

The recordings were contaminated with artifacts. The tABP values during the $72 \mathrm{~h}$ monitoring showed a skewed distribution in all the patients for all frequency bands and revealed a histogram with a long "right hand tail" representing extremely high values mostly found to represent artifacts (Fig. 1). All trimmed methods were compared with the visually edited method, which was used as a standard in this study (Fig. 2). Estimated marginal means with the $95 \%$ CI of the various differences showed NS difference between the visually edited and the $5 \%$ trimmed method in neither $\delta$-, $\theta-, \alpha$-, nor $\beta$-bands. The $10 \%$ trimmed method showed NS difference for the bands with low activity ( $\alpha$-and $\beta$-bands). All the other mathematically trimmed methods showed significant differences.

In this study, the 5\% trimmed method showed the closest relationship to the visually edited method and was used for further analyses of the two gestational groups. The demographic data of group 1 and group 2 are presented in Table 1 . The median of the tABP values showed a small asymmetrical distribution in both groups. Therefore, before analyzing the data a $\log$ transformation was done to reduce the skewness. The $\log$ transformed results $(\operatorname{lnt} \mathrm{ABP})$ are shown in Figure 3

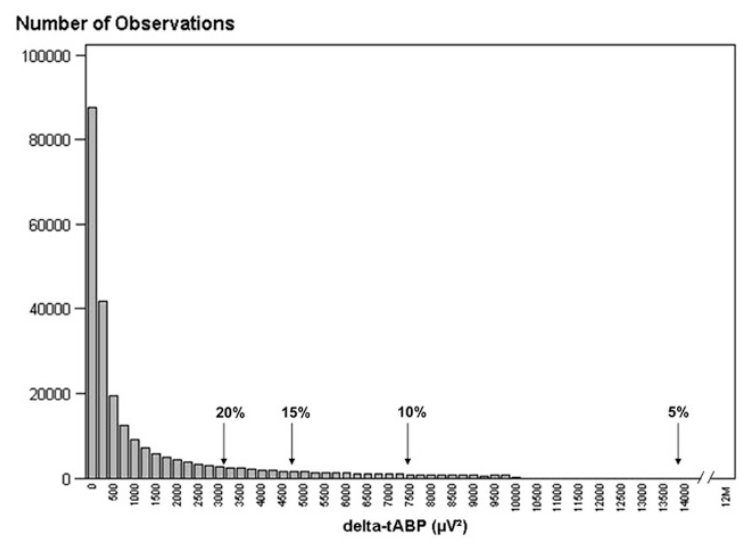

Figure 1. Histogram of the recorded $\delta$-tABP in one infant. It shows the skewed distribution of unedited data during 72-h continuous EEG monitoring with the "right hand tail" representing the high values of the artifacts. The arrows point to the cut-off values used for rejecting respectively $5,10,15$, and $20 \%$ of the data with highest power values as implemented in the algorithm used for trimming data. 

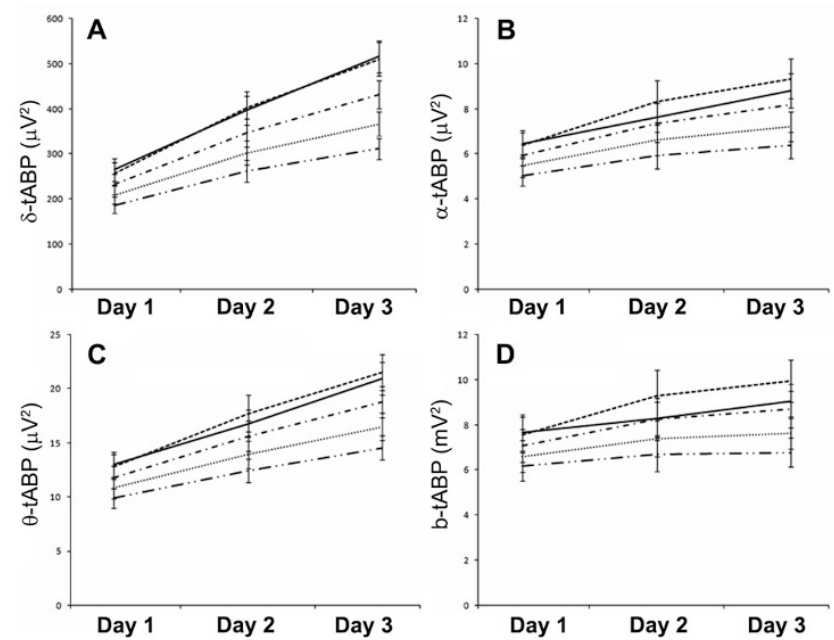

Figure 2. The $\mathrm{tABP}$ calculated as mean of the median \pm SEM in the different methods: the visually edited (solid line), 5\% trimmed (- - ), 10\% trimmed (-'--), 15\% trimmed $(\cdots \cdots)$, and $20 \%$ trimmed data $(\cdots \cdot \cdot \cdot \cdot) .(A)$ The $\delta$-band, $(B)$ the $\theta$-band, $(C)$ the $\alpha$-band, and $(D)$ the $\beta$-band. There was NS difference between the $5 \%$ trimmed and visually edited data in $\delta$-, $\theta-, \alpha$-, and $\beta$-bands and NS difference between the $10 \%$ trimmed and visually edited data in the $\alpha$ - and $\beta$-bands. The 15 and 20\% trimmed data differed significantly from visually edited data in all bands $(p<0.05)$.

Table 1. Demographic data of the healthy patient groups

\begin{tabular}{lcc}
\hline & Group 1 $(n=22)$ & Group 2 $(n=20)$ \\
\hline Male:female & $12: 10$ & $10: 10$ \\
GA $(w k+\mathrm{d})$ & $26+1[(24+2)-(27+5)]^{*}$ & $29+2[(28+0)-(30+6)]^{*}$ \\
Birth weight $(\mathrm{g})$ & $857(598-1138)^{*}$ & $1288(849-1836)^{*}$ \\
\hline
\end{tabular}

The population was divided into two groups according to GA.

* The values are presented as median (range).
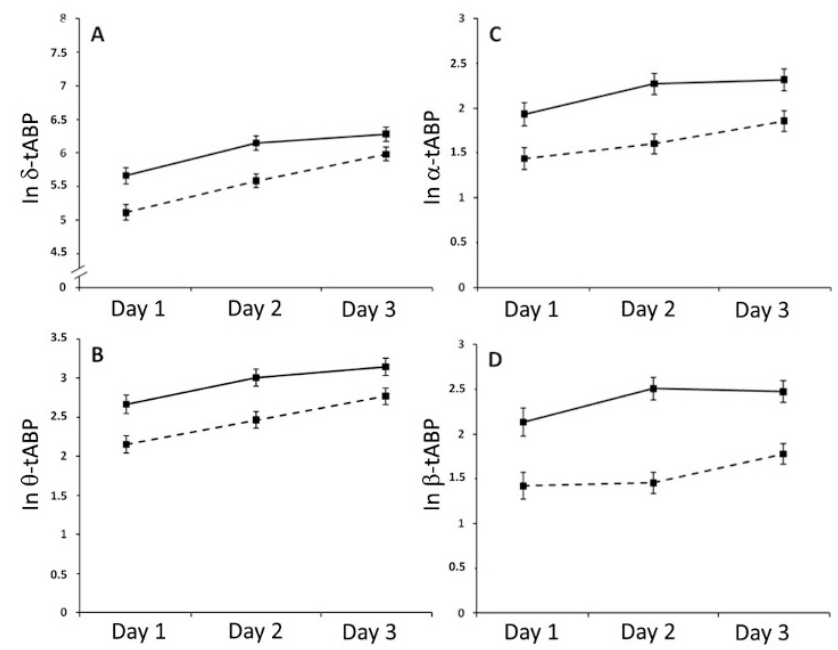

Figure 3. $\operatorname{lnt} \mathrm{ABP}$ of $(A)$ the $\delta$-band, (B) the $\theta$-band, $(C)$ the $\alpha$-band, and $(D)$ the $\beta$-band during the first $3 \mathrm{~d}$ of life (5\% trimmed method). Results are reported as natural logarithms (ln) of the median of absolute values expressed as mean \pm SEM. The discontinuous lines represent group 1 and the continuous lines group 2. There is a significant increase of tABP from $\mathrm{d} 1$ to 3 ( $p<$ $0.001)$ and a significant difference between the two groups for all the bands $(p<0.001)$. There is no significant difference in the increase of tABP from d 1 to 3 within the groups.

for both groups (the original tABP values are shown in Table $2)$. The lntABP showed a significant increase from $d 1$ to 3 in all the bands $(p<0.001)$. There was a significant difference in
Table 2. The values of the median of total absolute band power $(t A B P)$ from $d 1$ to 3 in the two groups

\begin{tabular}{|c|c|c|c|c|c|c|c|c|}
\hline & \multicolumn{2}{|c|}{ Delta } & \multicolumn{2}{|c|}{ Theta } & \multicolumn{2}{|c|}{ Alpha } & \multicolumn{2}{|c|}{ Beta } \\
\hline & $\begin{array}{l}\text { Mean } \\
\left(\mu V^{2}\right)\end{array}$ & SEM & $\begin{array}{l}\text { Mean } \\
\left(\mu V^{2}\right)\end{array}$ & SEM & $\begin{array}{l}\text { Mean } \\
\left(\mu V^{2}\right)\end{array}$ & SEM & $\begin{array}{l}\text { Mean } \\
\left(\mu \mathrm{V}^{2}\right)\end{array}$ & SEM \\
\hline \multicolumn{9}{|l|}{ Group 1} \\
\hline d 1 & 196 & \pm 29 & 10 & \pm 1 & 5 & \pm 1 & 6 & \pm 1 \\
\hline d 2 & 302 & \pm 46 & 13 & \pm 2 & 6 & \pm 1 & 5 & \pm 1 \\
\hline d 3 & 449 & \pm 48 & 18 & \pm 1 & 8 & \pm 1 & 7 & \pm 1 \\
\hline \multicolumn{9}{|l|}{ Group 2} \\
\hline d 1 & 323 & \pm 30 & 16 & \pm 2 & 8 & \pm 1 & 10 & \pm 1 \\
\hline d 2 & 512 & \pm 48 & 22 & \pm 2 & 11 & \pm 1 & 14 & \pm 1 \\
\hline d 3 & 577 & \pm 50 & 25 & \pm 2 & 11 & \pm 1 & 13 & \pm 1 \\
\hline
\end{tabular}
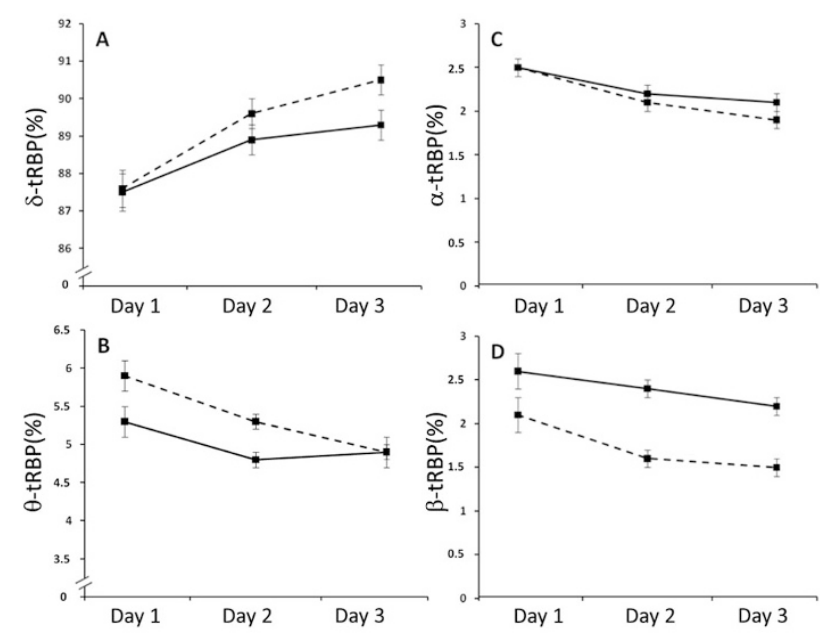

Figure 4. The median of RBP values (tRBP) expressed as mean \pm SEM of the different bands from $\mathrm{d} 1$ to 3 (5\% trimmed data). The discontinuous lines represent group 1 and the continuous lines group 2. (A) tRBP for the $\delta$-band shows a significant increase from d 1 to 3 in both groups $(p<0.001)$; tRBP for $\theta-, \alpha$-, and $\beta$-bands shown in $(B-D)$ demonstrate a negative correlation with time from $\mathrm{d} 1$ to $3(p<0.001)$. Except for the $\beta$-band, there is NS difference in tRBP between the two groups $(p<0.001)$.

tABP between group 1 and group 2 in all the bands throughout the study period $(p=0.001)$. In both groups, lntABP increased from d 1 to 3 . However, this increase did not differ between the two groups. The $\delta$-activity was the dominant EEG component followed by the $\theta$-activity, whereas the $\alpha$-and $\beta$-bands showed low activity during the first $3 \mathrm{~d}$. The tRBP showed significant differences from $\mathrm{d} 1$ to 3 in all the bands with an increase of $\delta$-tRBP and a decrease of tRBP of the other bands $(p<0.001$; Fig. 4$)$. For the $\delta$-, $\theta$-, and $\alpha$-bands, there was no correlation between tRBF and GA. Only the $\beta$-tRBP showed a significant difference between the groups $(p=0.001)$.

\section{DISCUSSION}

This is the first study that presents the feasibility of LTM with eight electrodes starting few hours after birth in extremely preterm infants from 24 wk GA. As the NICU staff gained more experience with the procedure throughout the study, it was possible to start the EEG monitoring closer to birth. It was performed without major interference with the daily routines. 
To find the most applicable electrodes was challenging. Seventy-two-hour continuous monitoring was intended per patient. The electrodes had to satisfy several requirements. They should be able to retain good contact during the whole study period, be resistant to the incubator humidity, and not be influenced by electrical interference. It was also important that they were well tolerated by the skin. Before starting the study, many types of electrodes were evaluated. With respect to prevention of painful procedures and the skin care of these immature infants $(19,20)$, needle electrodes were never considered. Only noninvasive electrodes (metal cup electrodes of two different sizes and different types of disposable electrodes) were tested. Blue Sensor BRS-50 K Ambu ECG electrodes were chosen as they fulfilled the criteria needed to get good long-term monitoring. Regarding the two patients excluded due to bad electrode contacts caused by hair, later experience has proved that the problem could have been solved by shaving the areas where the electrode should be placed. However, this was not possible due to the study protocol.

The handling of artifacts is a big challenge in the interpretation and quantification of the brain activity. Software with the function of automatic rejection of artifacts does not exist. To date, discrimination between artifacts and true biological activity mainly has been done by visual editing. The disadvantages of this method are that it is a time-consuming process and a subjective method strongly influenced by the interpreter (16). Trimming the unedited data by removing $5,10,15$, or $20 \%$ of the highest power values, respectively, may give a more representative sample of the true EEG activity. A more automated and objective type of revision would be preferable. In this study, the EEG results of visual editing were compared with the results of mathematically trimmed EEG data after removal of different percentages of the highest tABP values in each patient. The trends of automated trimmed data correlated well with the visually edited data. This supports our hypothesis that automatic trimming of EEG data is an applicable procedure in clinical settings.

Many methods and algorithms have been devised and applied in analyses of EEG monitoring. As FFT was among the first algorithms implemented in computers, many of the early methods of quantification of EEG build on FFT. Among these methods are spectral edge frequency (SEF) (21), ABP, and RBP. SEF defined as the frequency below which a certain amount $(50,90$, or $95 \%)$ of the power in the power spectrum resides was much used in previous brain monitors. It returned one number per time period, which made it easy to follow as a parameter of cerebral maturation. However, since Glaria and Murray (22) in 1985 declared that both a frequency-only technique and an amplitude-only technique could miss EEG changes, it was recommended to use a technique that encompassed both frequency and amplitude. In this study, both absolute and RBP were applied in the assessment of brain maturation.

An objection against FFT is the requirement of stationary signals, which is impossible to obtain when analyzing long-time epochs. This is taken into consideration by using short segments of $1 \mathrm{~s}$ and by implementing overlapping. By analyzing EEG of longer monitoring periods and calculate robust EEG parameters based on a big amount of assembled data, the effects of nonstationarities in the EEG recordings are minimized.

Another challenge in EEG monitoring is to discriminate between different activity states that affect the EEG activity. To our knowledge, no software for automatic analyses of sleep-wake cycling is available. Therefore, this still needs to be done visually. However, analyzing trends over time, it is the total brain activity that is expressed. Thus, discrimination between different brain activity states is not essential. We have shown that it is suitable to analyze LTM through an automated algorithm.

Because of the skewed distribution of tABP in each patient (Fig. 1), the median of tABP was used as a measure for the EEG activity per day. The trends of changes of tABP and tRBP d 1-3 are shown for infants born at 24-31 wk GA (Figs. 3 and 4). All frequency bands were analyzed as the early EEG activity from few hours after birth is not described in these infants yet. The results showed that tABP increases with GA for all the frequencies and support that the EEG activity is heavily dependent on GA (12). Furthermore, this study shows that the difference between GAs is present from the first day of life. The difference between the groups probably reflects increased arousal time and increased sleep-wake cycling in the more mature infants. The results are contrary to the findings in previous studies, which have reported a gradual decrease in the $\delta$ - and $\theta$-bands with increasing GA and no correlation between GA and maturational changes of the $\alpha$ - and $\beta$-bands $(13,14)$. These studies described EEG changes correlated to the EEG activity at postconceptional ages (PCA $=\mathrm{GA}+$ days after birth). However, a baby at 30 wk PCA may neurophysiologically differ from a preterm baby born at $30 \mathrm{wk}$ GA (23).

To our knowledge, this is the first study that demonstrates a changing EEG trend from d 1 continuing to $\mathrm{d} 3$. It was interesting to notice that $\mathrm{ABP}$ increased significantly during the first $3 \mathrm{~d}$ also in the more immature infants and that this increase did not differ from what was found in the more mature infants. The results of this study are partly in accordance with the study of Victor et al. (15) who performed an EEG spectral analysis during the first $4 \mathrm{~d}$ of life on premature infants from 24 wk GA. Their study showed an increase in ABP of the $\delta$-band over the first $3 \mathrm{~d}$ of life similar to our findings. However, in their study, ABP of the other frequency bands was unchanged in contrary to our findings. With regards to the RBP, both studies found that $\delta$-band increased, whereas $\theta-, \alpha-$, and $\beta$-bands decreased during the same time period. There was no relationship between RBP and GA for the $\delta, \theta$, and $\alpha$ in the two studies. This indicates that the change in the fraction between the frequency bands is similar in group 1 and 2. However, in our study, RBP showed a significant difference in the $\beta$-band between group 1 and 2 , which could indicate higher $\beta$ activity in group 2 (Fig. 4). The differences between these studies may be due to different monitoring time. Their monitoring time was short and not continuous and may not reflect possible changes in the same manner as a 72-h continuous monitoring.

In conclusion, this study demonstrates that early continuous EEG monitoring with eight electrodes is feasible in extremely 
premature infants starting soon after birth. This opens the possibility of continuous surveillance of the global brain activity during the first critical period of life. Furthermore, the study shows that mathematical trimming of EEG data matches the data achieved from visual editing. This demonstrates that visual editing of LTM may be substituted by mathematical trimming of the EEG data. This could be implemented in automated EEG analyses in preterm infants. We speculate that infants with brain pathology will deviate in their tABP trends from the normal tABP trend for their GA. In our study, one child was excluded because of IVH grade IV. This infant demonstrated a low tABP d 1-3. Although this is only one observation, it may support this hypothesis. Thus, in the future, LTM may be helpful to single out infants showing signs of brain pathology during the first days of life and may open for new treatment approaches.

Acknowledgments. We thank Professor Odd Aalen for statistical support, Hans Sjöholm, MD, PhD, for assistance with EEG interpretation, and the nurses in our NICU for their collaboration.

\section{REFERENCES}

1. Bhutta AT, Cleves MA, Casey PH, Cradock MM, Anand KJ 2002 Cognitive and behavioral outcomes of school-aged children who were born preterm: a metaanalysis. JAMA 288:728-737

2. Marlow N, Wolke D, Bracewell MA, Samara M 2005 Neurologic and developmental disability at six years of age after extremely preterm birth. N Engl J Med 352:9-19

3. West CR, Harding JE, Williams CE, Gunning MI, Battin MR 2006 Quantitative electroencephalographic patterns in normal preterm infants over the first week after birth. Early Hum Dev 82:43-51

4. Hellström-Westas L, Klette H, Thorngren-Jerneck K, Rosen I 2001 Early prediction of outcome with aEEG in preterm infants with large intraventricular hemorrhages. Neuropediatrics 32:319-324

5. Hellström-Westas L, Rosen I 2005 Electroencephalography and brain damage in preterm infants. Early Hum Dev 81:255-261

6. Hellström-Westas L, Rosen I 2006 Continuous brain-function monitoring: state of the art in clinical practice. Semin Fetal Neonatal Med 11:503-511
7. Biagioni E, Bartalena L, Boldrini A, Cioni G, Giancola S, Ipata AE 1994 Background EEG activity in preterm infants: correlation of outcome with selected maturational features. Electroencephalogr Clin Neurophysiol 91:154-162

8. Marret S, Parain D, Menard JF, Blanc T, Devaux AM, Ensel P, Fessard C, Samson-Dollfus D 1997 Prognostic value of neonatal electroencephalography in premature newborns less than 33 weeks of gestational age. Electroencephalogr Clin Neurophysiol 102:178-185

9. Selton D, Andre M, Hascoet JM 2000 Normal EEG in very premature infants: reference criteria. Clin Neurophysiol 111:2116-2124

10. Flores Guevara R, Giannuzzi R, Nosralla MO, Vignolo P, Moriette G, Maier MA 2008 Positive slow waves in the EEG of premature infants between 24 and 36 weeks of conceptional age. Clin Neurophysiol 119:180-189

11. Walls-Esquivel E, Vecchierini MF, Heberle C, Wallois F 2007 Electroencephalography (EEG) recording techniques and artefact detection in early premature babies. Neurophysiol Clin 37:299-309

12. Bell AH, McClure BG, McCullagh PJ, McClelland RJ 1991 Variation in power spectral analysis of the EEG with gestational age. J Clin Neurophysiol 8:312-319

13. Okumura A, Kubota T, Toyota N, Kidokoro H, Maruyama K, Kato T, Hayakawa F, Watanabe K 2003 Amplitude spectral analysis of maturational changes of delta waves in preterm infants. Brain Dev 25:406-410

14. Okumura A, Kubota T, Tsuji T, Kato T, Hayakawa F, Watanabe K 2006 Amplitude spectral analysis of theta/alpha/beta waves in preterm infants. Pediatr Neurol 34:30-34

15. Victor S, Appleton RE, Beirne M, Marson AG, Weindling AM 2005 Spectral analysis of electroencephalography in premature newborn infants: normal ranges. Pediatr Res 57:336-341

16. Niemarkt HJ, Andriessen P, Pasman J, Vles JS, Zimmermann LJ, Oetomo SB 2008 Analyzing EEG maturation in preterm infants: the value of a quantitative approach. J Neonatal Perinatal Med 1:131-144

17. Papile LA, Munsick-Bruno G, Schaefer A 1983 Relationship of cerebral intraventricular hemorrhage and early childhood neurologic handicaps. J Pediatr 103:273277

18. Ferree TC, Luu P, Russell GS, Tucker DM 2001 Scalp electrode impedance, infection risk, and EEG data quality. Clin Neurophysiol 112:536-544

19. Carbajal R, Rousset A, Danan C, Coquery S, Nolent P, Ducrocq S, Saizou C, Lapillonne A, Granier M, Durand P, Lenclen R, Coursol A, Hubert P, de Saint BL, Boelle PY, Annequin D, Cimerman P, Anand KJ, Breart G 2008 Epidemiology and treatment of painful procedures in neonates in intensive care units. JAMA 300:60-70

20. Darmstadt GL, Dinulos JG 2000 Neonatal skin care. Pediatr Clin North Am 47:757-782

21. Bell AH, McClure BG, McCullagh PJ, McClelland RJ 1991 Spectral edge frequency of the EEG in healthy neonates and variation with behavioural state. Biol Neonate 60:69-74

22. Glaria AP, Murray A 1985 Comparison of EEG monitoring techniques: an evaluation during cardiac surgery. Electroencephalogr Clin Neurophysiol 61:323-330

23. Conde JR, de Hoyos AL, Martinez ED, Campo CG, Perez AM, Borges AA 2005 Extrauterine life duration and ontogenic EEG parameters in preterm newborns with and without major US brain lesions. Clin Neurophysiol 116:2796-2809 